Journal of Biological Sciences, 5 (2): 114-118; 2005

ISSN 1727-3048

(C) 2005 Asian Network of Scientific Information

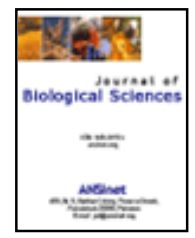

\title{
Comparative Oncology and Comparative Tumor Immunology
}

\author{
*Ivan Bubanovic and $* *$ Stevo Najman \\ *Department of Obstetrics and Gynecology - "Medica Centre" - Nis, Serbia and Montenegro. \\ **Institute for Biology, University Medical School - Nis, Serbia and Montenegro.
}

\begin{abstract}
Comparative oncology is a branch of comparative pathology that is relatively new biomedical discipline. The need for a comparative research into tumors across different groups of living beings has arisen from a relatively old notion that most of multicellular organisms may develop tumors. This apparently very simple fact indicate the possible nature of tumor growth, which can be associated with the basic features of the cells of multicellular organisms such as the division, development, growth and differentiation of cells and tissues. Regarding this, tumor growth is commonly defined as a fundamental disorder in the regulation of cell division, growth, differentiation and cell socialization. In all vertebrates, neoplasia is a disease in which genetically altered cells escape from the normal cell-cycle regulation and monitoring of the immune system. This results in a persistent, expanding or infiltrating growth without control and the architecture of the normal tissue. Concerning the facts that mechanisms of cell sociability control and anti-tumor immune monitoring might be different in different group of animals, especially, in different classes of vertebrates, development of disciplines such as comparative oncology and comparative tumor immunology can be of great scientific importance.
\end{abstract}

Keywords: vertebrates, immunity, tumor, viviparity, evolution

\section{COMPARATIVE ONCOLOGY}

One of the tasks of comparative oncology is the identification of carcinogenic factors and mechanisms of their influence on the cells of different, phylogenetically distant multicellular organisms, as well as of differences in sensitivity of these cells to carcinogenic factors. Even though there are no reliable data regarding the incidence of tumors in different groups of multicellular organisms, some studies indicate the possibility that tumor incidence might be growing along with the growing complexity of observed species and their place on the evolutionary scale. ${ }^{[1,2,3,4]}$

The rationale behind such phenomenon could be the stability of genome and the reliability of mechanisms controlling the cell-cycle. For example, simple multicellular organisms, such as yeasts, may have simpler and more reliable machinery and more effective repair mechanisms for damaged DNA sequences. Unlike these, higher multicellular organisms, like vertebrates, have a more complex but less stable genome, as well as more complex surveillance mechanisms, which could be taken into account for a higher tumor incidence in vertebrates in comparison with the lower multicellular organisms. Yet, there are data that significant differences regarding tumor incidence might be present across different classes of vertebrates.

Thus, Effron et al. ${ }^{[5]}$ presented the rate of neoplasia at necropsy of captive wild animals of the Zoological Society of San Diego collection. Neoplasia was present at necropsy in $2.75 \%$ of 3,127 mammals, $1.89 \%$ of 5,957 birds, and $2.19 \%$ of 1,233 reptiles. Interestingly, neoplasms were not detected during 198 necropsies of amphibians. The same authors argue that the most common types of tumors differ greatly across vertebrate classes as well ${ }^{[5]}$. Notably, lymphosarcoma was the most common tumor registered in birds and reptiles while various types of tumors, such as adenomas, hepatoma and different lines of carcinomas were registered in mammals.

Coresponding Author: Dr. Ivan Bubanovic, Ob/Gyn Department - "Medica Centre", Novosadska 1/c, 18000 Nis, Serbia and Montenegro, Tel: (+381)18249178, E-mail: ibubanovic@yahoo.com 
Unfortunately, the research did not include tumor incidence in cartilaginous fish and bony fish, though there are data indicating a very low tumor incidence in the former and particularly in the latter. Namely, Hendricks et al. ${ }^{[6]}$ failed to prove the presence of tumors on 144 necropsies in brown bullheads (Ictalurus nebulosus), but tumor incidence in the same type of fish taken from polluted waters ranged $30 \%$ on the sample of $532^{[6]}$.

Neoplasms do appear in all non-mammalian vertebrates and this is important in and of itself since these animals can serve as models to understand the behaviour and trajectory of such tumors in mammals and humans. Thus, an inter-disciplinary effort must begin among collectors, zookeepers, veterinarians, comparative pathologists in order to obtain comprehensive documentation that would help us to understand neoplasms more thoroughly not only in non-mammals, but also in mammals and humans.

There are many reasons to believe that the phenomenon of carcinogenesis follows the same or similar postulates across all vertebrate classes. A large number of studies also indicate the possibility that the biological fundaments of carcinogenesis in vertebrates are observable in invertebrates, as well. It is clearly, therefore, that one of the primary steps leading towards carcinogenesis in all vertebrates is the destabilization of the genome and loss of cells' ability to repair DNA damage. Genome destabilization ending in carcinogenesis is, basically, the consequence of a series of mutations or translocations, and comes largely as a result of the influence of chemical agents, viral infections, radiation and chronic inflammations including auto-immune processes. What makes an altered cell malignant is the activation of individual genes or group of genes, otherwise normally functional during embryogenesis. An incomplete and inadequate ontogenic regression in terms of both time and space results in the loss of sociability of the altered cell, destruction of its environment and the host. The described mechanism of cell alteration has been verified in all classes of vertebrates, except for cartilaginous fish where few cases of tumors have been registered. Some of the reasons for considerable variations regarding tumor incidence across vertebrate classes, may be contained within genome stability, as well as various possibilities for repairment of the DNA damage ${ }^{[7,8]}$.

Although some neoplasms are directly hereditary, genetic predisposition is only one of factors affecting the occurrence of all neoplasms. The tendency of certain species to develop particular types of tumors is a well-known aspect of comparative oncology [9]. Apart from a great similarity in cell organization across vertebrates, there is an opinion that DNA and mechanisms regulating the cell-cycle in lower vertebrates are more stable and more resistant to the influence of various carcinogens ${ }^{[10]}$. This explanation is quite acceptable from the aspect of control mechanisms of the cell-cycle and DNA stability as being "the first line of defence" from malignant cell alteration. The immune system could represent "the second line" of anti-tumor defence. Due to the possible significance of the immune system in anti-tumor protection, differences in anti-tumor efficacy among different groups of multicellular organisms could also be of great influence for the incidence of manifesting tumors ${ }^{[4]}$. However, it is very difficult to make a viable conclusion as to how much the failure of the "first", particularly "the second" line of defence really contribute to even greater differences in tumor incidence across various groups of multicellular organisms. This dilemma may be solved by future research in the field of comparative oncology, particularly by developing disciplines such as comparative tumor genetics and comparative tumor immunology.

\section{ANTI-TUMOR IMMUNITY FAILURE IN MAMMALS}

In the theory, anti-tumor immunity in invertebrates might be based solely on the activity of the innate immune system, relatively poorly developed network of molecular mediators, a small number of effector cells and proportionally poorly developed mechanisms regulating immune response. Therefore, one of the primary tasks of comparative tumor immunology may be the identification of mechanisms of anti-tumor immunity in invertebrates and comparing them with their counterparts in vertebrates. On the other hand, all vertebrates are characterized by both innate and adaptive immunity, displaying great differences related to the advancement stage of the adaptive immunity, its correspondence with innate immunity. In addition, differences in the mechanisms controlling the immune reaction, number of cytokines and their role in the immune response are also important. The identification and comparative analysis of the foregoing mechanisms in invertebrates and vertebrate classes may contribute significantly in clarifying the mechanisms of anti-tumor immunity failure in mammals, as well as tracing the possible link between these and immunoreproductive mechanisms. 
Furthermore, this could be a way of introducing new, more effective adjuvant and/or immunotherapeutic procedures ${ }^{[4,11]}$.

Based on recent findings, several mechanisms have been identified that participate in the development of the phenomenon of anti-tumor immunity failure in vertebrates:

1. The processes of embryogenesis and mechanisms establishing central immune tolerance to "self" molecules are similar across all vertebrate classes. Therefore, the mechanisms of immunotolerance to embryonic and/or embryonic-like tissues, such as tumor tissues, may be connected with central immunotolerance ${ }^{[11,12]}$. There is evidence that the mechanisms of central immunotolerance are, more or less, active in adults. This phenomenon could also be involved to some extent in the development of antitumor immunity failure ${ }^{[12,13]}$;

3. The mechanisms of peripheral maturation of lymphocytes and possible influence of these on the quality of the immune reaction have been also verified in most vertebrate classes ${ }^{[13]}$;

4. The immune system of vertebrates is closely connected with auto-immunity being a by-product of the adaptive immunity. "Self"-protective immune reaction control mechanisms that could become activated in anti-tumor immune reaction, co-evolved alongside auto-immunity as a "new" evolutionary phenomenon and a new form of selection pressure. Therefore, the activation of anti-tumor immunity as a unique form of auto-immune process was probably followed by a parallel activation of protective mechanisms, i.e. the immune reaction control mechanisms ${ }^{[4]}$;

5. Unlike non-mammals, the immune system of mammals has "built-in" the mechanisms of tolerance to proliferative tissues like trophoblast. These mechanisms have developed, in the course of evolution, under a very strong selection pressure of alloimmunity and reproductive efficacy. Although very similar to the mechanisms resembling autoimmunity, the mechanisms of immune tolerance to trophoblast can be regarded as more advanced and more effective. On the other hand, there is a great similarity between the mechanisms of immune tolerance to trophoblast and anti-tumor immunity failure mechanisms. These two, apparently diverse mechanisms may be regarded as a protective immune cross-reaction against the proliferative tissues of diametrically different origin ${ }^{[4,11]}$.

All vertebrates are, more or less, susceptible to carcinogenesis, depending on the sensitivity of their
DNA to the influence by various carcinogenic factors. The phenomenon of anti-tumor immunity failure is relatively easily verifiable in all vertebrate classes, due to the similarities in organization and functioning of their immune systems. The phenomenon of anti-tumor immunity failure in non-mammals rests largely on the mechanisms of central immune tolerance to embryonic and/or embryonic-like cells and control mechanisms resembling auto-immunity. However, the diversification of the immune reaction control mechanisms in mammals has again produced new possibilities regarding tolerance to proliferative tissues, i.e. trophoblast and tumors. Several observations could be taken as a basis for the future research in the field of comparative tumor genetics, immunology and immunogenetics:

1. The expression of class I and class II molecules, tissue distribution of the molecules and level of polymorphism of class I and class II genes in non-mammals and mammals are substantially different. In most non-mammals, class II genes are more polymorphic in relation to class I, while class I genes are highly polymorphic in mammal genome. Tissue distribution of class II molecules in mammals is restricted on APCs, dendritic cells and B lymphocytes, while non-mammals shows the phenomenon of poor restricted or unrestricted tissue distribution of the class II molecules ${ }^{[14,15]}$.

2. In all non-mammalian classes of vertebrates class I and class II genes are rambling through genome, but LMP and TAP genes are highly evolutionary conserved within class I region. In mammals, class I and class II genes are clustered on the same chromosome (except equine), but LMP and TAP genes are conserved within class II region ${ }^{[16,17,18]}$.

3. The transcription of class II/LMP/TAP genes in mammals are controlled from same signals. The absence of class II genes transcription signals lockout antigens processing machinery and class I molecules peptide presentation, as well as the activation of Th1 cells and adaptive immunity effectors actions ${ }^{[19,20]}$. In non-mammals, antigen processing machinery is under control of class I genes transcription, because class I/LMP/TAP genes are closely connected on the same chromosomal loci ${ }^{[16,17,18]}$.

4. Anti-tumor immunity in non-mammalian (except birds) vertebrates predominantly depend on the innate immune system, while anti-tumor immunity in mammals depend on the innate and adaptive immune systems and their communication ${ }^{[21]}$.

5. The specificity in expression and tissue distribution of MHC genes, LMP and TAP genes 
transcription control, as well as the communication between native and adaptive immunity in nonmammalian vertebrates qualifies a substantially different cytokine network and immune reaction than in mammals.

6. There is a possibility that malignant cells in fishes, amphibians, reptiles and birds are more susceptible to apoptosis than mammalian malignant cells ${ }^{[22]}$.

7. The high resistance on carcinogens induced genetic changes is evidenced in some experiments with lower vertebrates, leading to a conclusion that DNA from lower vertebrates shows a high level of resistance on carcinogenesis ${ }^{[2,22]}$.

8. The complex and efficient mechanisms of immune reaction control developed under the evolutionary pressure of high polymorphism of class I genes, auto-immunity and reproductive effectiveness can be included in the mechanisms of anti-tumor immunity failure in mammals.

9. Mammalian extended cytokine network can be activated/deactivated by same or similar factors under different conditions such as pregnancy and malignancy. A small number of cytokines and poor cytokine network are the characteristics of nonmammalian vertebrates. For example, cytokines like IL-10 and IL-4 are unknown in fishes and amphibians, but TGF- $\beta$ is evidenced in reptiles, birds and probably in other non-mammalian classes ${ }^{[23,24]}$.

10. Th-like cells are detected in reptiles and amphibians ${ }^{[25]}$, as well as Th and/or Th1-like cells in birds ${ }^{[26]}$, but mammals are single vertebrates which have the advanced system of immune reaction control established on Th1 and Th2 cells, and their balanced activity. The absence or fractional awareness of $\mathrm{Th} 2$ model of immune reaction control probably contributes in the strong anti-tumor immunity in nonmammalian vertebrates.

11. Mammalians' immune system may be tolerant to cancer cells because they are very similar to trophoblast cells ${ }^{[11]}$.

12. Sex hormones, steroids and other factors, which are the attributes of pregnancy and malignant processes, can impair blood-thymus barrier. It can be another mechanism of acquired thymic tolerance to foreign molecules in pregnancy and malignancy ${ }^{[12]}$.

13. The absence of MHC and costimulatory molecules expression, prostaglandine, Th2 cytokines, sex hormones, steroids and other factors could be promoter of extrathymic lymphocytes maturation in antigen-protective manner in mammalians. It is yet one of the mechanisms that are included in trophoblast and tumor escape ${ }^{[13]}$.

14. Unlike mammals, the mechanisms of immune reaction control in non-mammalian vertebrates probably are essentially independent from an important role of co-stimulatory molecules. Actually, co-stimulatory molecules like CD40, CD80, CD86 and OX40 were not detected in non-mammalian vertebrates, except CD80 and CD86-like molecules in birds ${ }^{[27]}$.

\section{CONCLUSION}

The biological phenomena whose mechanisms are, at present, explored and largely understood, certainly had their own evolution. Searching for the origin and details of the evolution of "advanced solutions" as well as selection pressures that might justify their emergence and existence, we often fail to see that many such phenomena are, in fact, co-evolutionary byproducts of "evolutionary innovations". In other words, the evolutionary emergence of "advanced solutions" is sometimes, if not always, accompanied by certain by-products and by the co-evolution of compensatory mechanisms acting as a counterbalance to these. An example of the evolution of "advanced solutions" is the evolution of adaptive immunity, and co-evolution of auto-immunity and alloimmunity. Alongside with the diversification of the mechanisms of adaptive immunity, auto-immunity and alloimmunity gain attribute of the evolutionary byproducts and become sources of selection pressure. To that effect, alloimmunity could be a source of very strong selection pressure in mammals, simply because it is directly connected with the reproductive efficacy. At the same time, new forms of selection pressure that are connected with adaptive immunity gave rise to new mechanisms controlling killer machinery of the immune system. Finally, the last in a line of byproducts in the processes of evolutionary "modelling" and "re-modelling" of vertebrate immune system can be called the failure of anti-tumor immunity.

\section{REFERENCES}

1. Stewart H.L. (1972). Cancer and comparative pathology. Prog Exp Tumor Res. 16:142-150.

2. Harshbarger J.C. (1974). Activities Report Registry of Tumors in Lower Animals, 1965-1973. RTLA 1385. Smithsonian Institution Press, Washington, D.C.

3. Harshbarger J.C. (1996). Comparative oncology. Jpn J Cancer Res. 87:1-6. 
4. Bubanovic I., Najman S. (2004). Anti-tumor Immunity Failure in Mammals - Evolution of the Hypothesis. Acta Biotheor. 52:57-64.

5. Effron M., Griner L., Benirschke K. (1977). Nature and rate of neoplasia found in captive wild mammals, birds, and reptiles at necropsy. J Natl Cancer Inst. 59:185-198.

6. Hendricks J.D., Sinhuber R.O., Loveland P.M. et al. (1980). Hepatocarcinogenicity of glandless cottonseeds and cottonseed oil to rainbow trout (Salmo gairdnerii). Science. 208:309-311.

7. Schwemmler J.W. (1991). Carcinogenesis as reversal of eukaryotic symbiogenesis: The aposybiosis thory of cancer endocytobiosis. Cell Res. 7:163-199.

8. Schwemmler J.W. (1998). Basic Cancer Programs Genes, Signals, Metabolites - Unified Holistic Theory of Evolution. Karger.

9. Shlumberger H.G. (1957). Tumors characteristics for certain animal species. Canc Res. 17:823-832.

10. Ruben N.L., Clothier H.R., Balls M. et al. (1997). Cancer resisitance in amphibia. Dev Comp Immunol. 21:102-102.

11. Bubanovic I. (2003). Origin of Anti-tumor Immunity Failure in Mammals and new Possibility for Immunotherapy. Med Hypotheses. 60:152-158.

12. Bubanovic I., (2003). Failure of blood-thymus barrier as a mechanism of tumor and trophoblast escape. Med Hypotheses. 60:315-320.

13. Bubanovic I. (2003). Crossroads of extrathymic lymphocytes maturation pathways. Med Hypotheses. 612:235-239.

14. Hughes A.L., Nei M. (1993). Evolutionary relationships of the classes of MHC genes. Immunogen. 37:337-342.

15. Lawlor D.A., Zemmour J., Ennis P.D., et al., (1990). Evolution of class-I MHC genes and proteins: from natural selection to thymic selection. An Rev Immunol. 8:23-29.

16. Kasahara M., Hayashi M., Tanaka K., et al. (1996). Chromosomal localization of the proteasome $\mathrm{Z}$ subunit gene reveals an ancient chromosomal duplication involving the major histocompatibility complex. Proc Nat Acad Sci USA. 93:9096-9101.

17. Kasahara M., Nakaya J., Satta, Y. et al. (1997). Chromosomal duplication and the emergence of the adaptive immune system. Trend Gen. 13:90-92.

18. Kaufman J., Wallny H.J. (1996). Chicken MHC molecules, disease resistance and the evolutionary origin of birds. Cur Top Microbiol Immunol. 212:129-141.

19. Chaux P., Moutet M., Faivre J. et al. (1996). Inflammatory cells infiltrating human colorectal carcinoma express HLA class II but not B7-1 and B7-2 costimulatory molecules of the T-cell activation. Lab Invest. 74:975-983.

20. Paul P., Rouas-Freiss N., Khalil-Daher I. et al. (1998). HLA-G expression in melanoma: A way for tumor cells to escape from immunosurveillance. Proc Nat Acad Sci USA. 95:4510-4515.

21. Robert J., Cohen N. (1999). Evolution of immune surveillance and tumor immunity: studies in Xenopus. Immunol Rev. 166: 231-243.
22. Laurens N.R. (1997). Cancer resistance in amphibia. Dev Com Immunol. 21:102-106.

23. Paulesu L. (1997). Cytokines in mammalian reproduction and speculation about their possible involvement in nonmammalian viviparity. Micros Res Tech. 38:188-194.

24. Reboul J., Gardiner K., Monneron D. et al. (1999). Comparative genomic analysis of the IFN/IL-10 receptor gene cluster. Gen Res. 9:242-250.

25. Wei Y.Q., Huang M.J., Yang L., et al. (2001). Immunogene therapy of tumors with vaccine based on Xenopus homologous vascular endothelial growth factor as a model antigen. Proc Nat Acad Sci USA. 98:11545-1150.

26. Vandaveer S.S., Erf G.F., Durdik J.M. (2001). Avian T helper one/two immune response balance can be shifted toward inflammation by antigen delivery to scavenger receptors. Brit Poul Sci. 80:172-181.

27. O'Regan M.N., Parsons K.R., Tregaskes C.A. et al. (1999). A chicken homologue of the co-stimulating molecule CD80 which binds to mammalian CTLA-4. Immunogen. 49:68-71. 